\title{
Curricular Integration in Each Area of Learning and the Acquisition of Other Areas of Learning
}

\author{
Nereida Balla \\ Phd. Candidate \\ Email: balla.nereida@yahoo.com
}

\section{Doi:10.5901/mjss.2016.v7n4p}

\section{Abstract}

The curriculum is understood as a system composed of various interconnected educational goal elements, enabling the social occasion of training organization through training and administrative programs. Curricular reform presents a number of challenges to the Albanian context: Foremost, the conversion from a behavioral approach to a constructive approach will require its time and intensive work of MAS and all the teaching institutions in Albania to train teachers working in the preuniversity system. On the other hand, this new challenge in the Albanian education requires cooperative partners such as Albanian Faculties of education, in society to place the emphasis of competence-based approach in their academic course of studies. Together with the changes taking place in our society, new requirements, new relationships in a social environment and labor market, technological innovation and new human aspirations arise, as well. With the myriad numerous fast changes, quantitative and qualitative in all areas of life, our country is currently crystallizing the content of curriculum reform, as one main direction of the reform. The study attempts to answer the following questions: (Pedagogical magazine "Education Review" Tirana 2014): - Is integrated curriculum an answer? - Is it a trend in our education? - Do students really benefit from integrated curriculum? - What are the contemporary approaches to curriculum development? - What kind of problems do teachers face when implementing the curriculum?

Keywords: curriculum integration, the fragmented curriculum, cross-curricular accomplishments, personality of the pupils, coordination of fabrics.

\section{Characteristics of the new course of study}

Whenever made efforts to define an integrated curriculum is necessary to carefully understand the terms employed. Single of the most accepted definitions today is that of Humphreys: according to him integrated curriculum is:

"An integrated work in which students widely explore knowledge in several fields, nearly connected to certain faces of their surroundings" ( J.R. Humpreys.)

Thus this author examines the ties between the social sciences, communication arts, science, mathematics, art and music. According to him, knowledge and skills are evolved and implemented in more than one field of disciplines.

Hereinafter in this determination, Shoemaker defines the integrated curriculum as:

"Education organized on-linking of different events that put different aspects of the curriculum in common background of the relations between different fields of knowledge. In this way of memorizing and teaching, it is the most comprehensive way which reflects the interconnections that exist in the material world ". Integrated curriculum, the planned experience and learning, not only supplies the scholar with a unified aspect of knowledge acquired (through the acquisition of models, schemes, cultural structures, etc.), but also promotes and develops the student's power to capture and interpret the new relationship, therefore, the ability to create models, new schemes and constructions. Another term commonly employed in expressing the integrated curricula is inter-subject curriculum by which the organization of curricula is seen based on logging point of learning areas and targets to concentrate attention to the comprehensive understanding of life problems and the origination of more broad fields of cognition that work various aspects of the course of work in their meaningful connections.

Under this view, inter-subject curriculum is viewed in a single project coordination of disciplines or different groups of subjects at school.

Casting the theory with the real school curricula together, is a significant step in school arranging programs. Not all the theories are indulgently assessed in actual pattern. Society development as a knowledge and global one has contributed to different opinions on the importance of knowledge, it has increased attention on the importance of competence and has also ordered changes in the design of curricula. The new curriculum paradigm has not foreseen 
replacing the existing curriculum with a new one, but to re-dimense, modify, and reconstruct it.

All the definitions given for integrated curriculum are grounded along the belief that:

"The school must evaluate education as a cognitive operation that aspires to develop the skills needed in today's universe, away from restrictions offering a fragmented curriculum. "

In general, all the definitions of integrated curriculum or cross-curricular curriculum include:

- Co-ordination of cases

- Greater emphasis on task work

- Sources of info or knowledge beyond textbooks

- Relations between concepts

- Thematic units as organizational principle

- Non solid mappings

- Interchangeable counter students

\subsection{Powers in Albania curricular framework (Pedagogical magazine Tirana 2011)}

After reading some of the curriculum competencies in Europe and worldwide, in appropriate with the targets of preuniversity education, it has been indicated that key competencies for further development of Albania education area:

1. Competence of communication and reflection;

2. Competence of thinking;

3. The competence of learning to read;

4. Responsible for life, entrepreneurship and the surroundings;

5. Personal jurisdiction;

6. Civil competence;

7. Digital competence.

Construction of pre-university teaching along with these seven competences will create the possibility of fulfillment of demands for curricular changes and help the constitution of a propagation of immature people capable to apply knowledge in a more hardheaded.

\section{Cross-curricular accomplishments (Pedagogical magazine Tirana 2011)}

Pre-University education curriculum aims to develop these cross-curricular accomplishments:

\subsection{Ability of communication (Pedagogical magazine Tirana 2011)}

Communication, in the sense of granting and taking in information, ideas and images, transmission and feelings, are developed for students in each of the regions of learning.

Curricula must give each pupil the opportunity to be able to communicate clearly, accurately and understandably in diverse ways. (Pedagogical magazine "Education Review" Tirana 2014)

It should aspire to enable students to the standards of education levels: the transmit orally and in penning, in accordance with different situations, information, thoughts, opinions and images correctly, utilizing an increasingly richer vocabulary: -To recognize, examine and interpret texts and diverse patterns of communication; -to express their opinions, discuss, debate and engage in debate; -To dialogue, listening attentively, promoting and respecting the feelings of others.

\subsection{The ability of critical thinking (Pedagogical magazine Tirana 2011)}

Critical thinking ability enables the increase in the level of arguing and independent justification of students. Critical thinking requires the collection, selection of necessary data, assessment and reaching a stopping point.

The curriculum aims to enable students:

-To use various methods, to identify the structures of information and ideas

-To support with arguments and facts beliefs, generalizations, and their conclusions

-To keep or create independent attitudes, supported with arguments against the opinions and attitudes of others;

-To explain the associations between the whole integral parts, separate facts from readings and produce courses of logical thinking based on facts / findings / projections;

-To be able to view and adjust themselves. 


\subsection{Ability of creative thinking}

Creative thinking enables the discovery of raw ideas, creation of original connections among them and encountering a variety of responses to problematic situations in whole areas of scholarship, the curriculum enables students to exhibit originality and become their creative inclinations. It aspires to enable students: ( Pedagogical magazine "Education Review" Tirana 2014 )

-To express, through a diversity of forms, original personality sides rebelled in school, community and workplace;

-To assume and look for new breakthrough idea;

-To welcome new ideas and experiences of others;

-To furnish opportunities for misunderstandings and failures and determine resolutions to avert them.

\subsection{Ability of solving the problem}

To resolve a problem situation means to utilize the knowledge acquired to solve a previously unknown place. Problem solving ability gives students the opportunity to challenge, to solve the problem manage diverse situations of daily life, enabling them to simultaneously detect and investigating the scientific and technical world. It functions as the foundation for further learning and prepares the individual to be active and effectual in their own spirits, as considerably as in the social. In this respect, the curricula of secondary education aim to enable students:

- Analyze problem situation and formulate programs to solve their interoperability;

-To trace and measure the performance of stages to resolve the trouble;

-To seek, to obtain different solutions and judge them according to the usefulness and applicability;

-To measure the resolutions in order to ameliorate them;

-Communicate the solution to others.

\subsection{Ability to use and processing of information and communication technologies (ICT)}

Use and processing of information help students become more effective in an environment overflowing with information. For this reason the curriculum of pre- university education aspires to enable students:

-To supply and select data from several informants and in conformity with their design;

-To use techniques and strategies of various forms, with the purpose of obtaining, analyzing, processing, and interpretation of information;

-To judge the reliability and credibility of sources, using measures such as: authorship, bond, currency, experience of the author;

-To provide information, using a variety of information and communication technologies (e.g, Power Point, TV clips, and camera, digital).

\subsection{Ability to work in a group}

The scholar should take advantage of curricula ability to work in a group with others, in different environments and circumstances and with clear ends. This ability implies that he will be able to convey ideas and needs within the group, respecting different views of the other members, to reach agreement, to assume its responsibilities as a member of the group or its leader, to help others feel involved in the group and motivate it and force the group to overcome troubles.

\subsubsection{The curriculum aims to enable students:}

-To forge with others in a group, in different environments and contexts, with defined goals and aims;

-To communicate ideas and needs within the group, respecting different perspectives of its members;

-To achieve agreement on action plans to satisfy the deadlines and plans accepted;

- To take over the obligations of being part of the group as a member or as its drawing card;

-To help others feel included in the group and motivate the group to overcome troubles.

\subsection{Ability of social and ethical posture}

The school aspires to develop the general values and principles recognized by the society, which are linked up to the 
protection of human rights and children in particular, the respect and the maturation of internal values, the protection of the environment in all its diversity, sustainable development and so on

In all areas of scholarship, the curriculum enables pupils to uphold an ethical and societal attitude to events, phenomena behaviors and different views in society.

\subsubsection{It aims to enable students:}

- To assess the behaviors, actions and attitudes and others from the standpoint of the common good of the community, the nation and beyond;

- To evaluate the phenomena, events, attitudes and actions of others in the past and in the present in terms of social benefit to the community, the nation, the region and beyond;

- To be active participants in activities directed at community-scale improvements, the country, the region and beyond.

\subsection{The context of the curriculum}

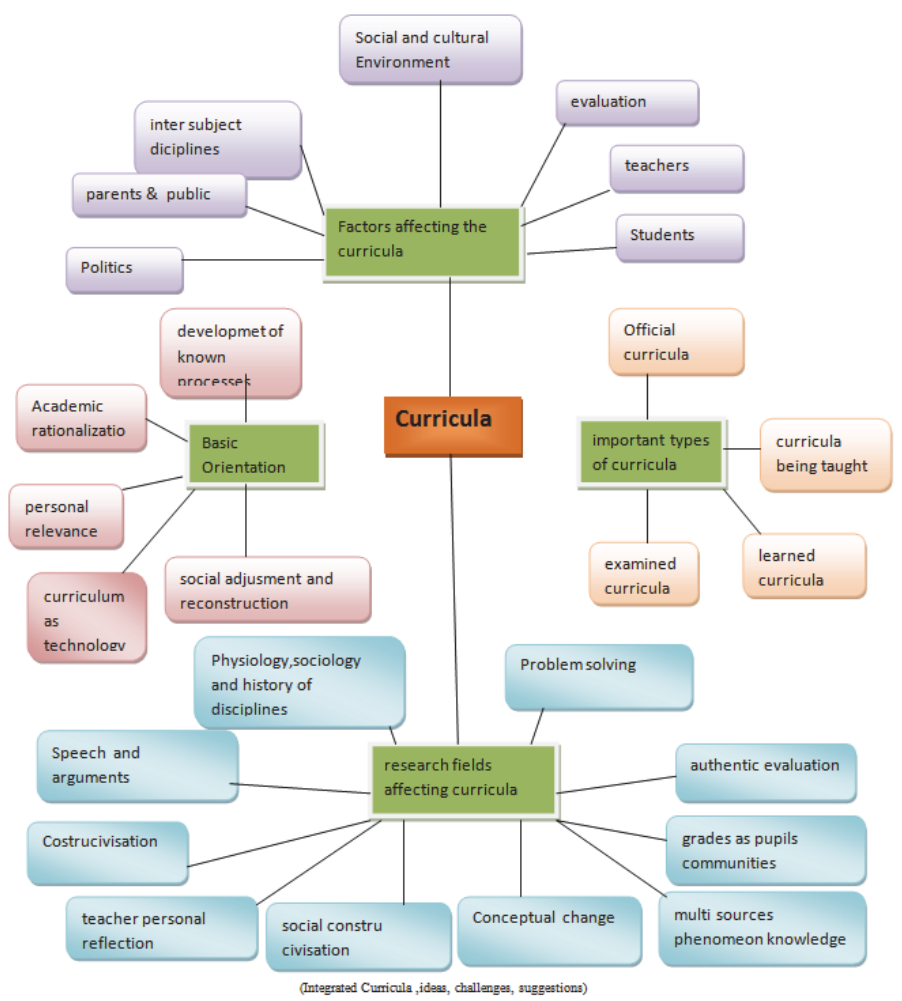

\section{Examples of application of global education in different subjects through fusion model.}

\subsection{Albanian language}

- Using literature to explore universal themes (conflicts, justice, love, etc.)

- Review of historical changes in terminology and linguistic variations associated with ethnicity, culture, gender. . (Integrated Curricula, ideas, challenges, suggestions)

- Developing awareness of the attitudes, beliefs, viewpoints and personal values; match different aspects and positions. 
- Develop interpersonal communication skills and empathic understanding through game play or dramatic play; (Integrated Curricula, ideas, challenges, suggestions)

- Reading of works from different states and cultures of the world;

- Identify stereotypes, prejudices and distortions through analysis of different works and textbooks;

- Identify the implications, biases, values, and so on through comparisons with the written form and television; (Integrated Curricula, ideas, challenges, suggestions)

- Exploring the significance of non-verbal communication in other cultures and in the home.

- Exercise the skills of communication, negotiation and examination through involvement in group discussions;

- The development of presentation skills of oral and written ideas by engaging in meetings with politicians etc., for local issues and global importance.

\subsection{Mathematics}

- Using statistics from real life data as the basis for practicing math skills;

- Developing skills, for model purpose, forecasting using global statistics on birth, death, growth and use of natural resources;

- Exercise basic skills of counting using systems and techniques from different cultures;

- Explore geometric shapes and patterns, studying art and trends in different civilizations;

- Analysis of the price and nutritional value of some wares and the planning of a menu not only healthy but also affordable;

- Exploring the concept of exponential growth on the level of interest and data on world population; (Integrated Curricula , ideas, challenges, suggestions)

- Working in groups to design alternative proposals, including budgets and timelines for the naturalization of the school environment.

\subsection{Science}

- Understanding of ecological principles by going over the ecosystem, energy flow, cycles and food chains;

- Studying the chemical science of atmospheric and water contamination and the monetary values and benefits of measures for its liquidation;

- Review the impact of coal mining on human health etc. and other natural species and ecosystems;

- Taking into account the advantages and disadvantages of alternative means of energy output;

- Design and construction of a model wind generator or water pump for use in growing nations;

- Using imagination techniques to ease understanding of the water wheel and to undertake an imaginary journey to the universe of atomic weapons;

- Analysis of the usage of technology in contemporary life and forecast technological preferable future

- Forecast the impact of global warming or the ozone hole in the different species and their habitat;

- Exploring the impact of malnutrition and abusive substances on human wellness,

- Art. Magical wealth forms of nature is an important source for the unfolding of imagination and creation in the field of art, giving a valuable contribution in shaping the aesthetic of students through music, painting, art activities, etc. .

- Language. Chemistry program requirements 9 in connection with the description, understanding and prediction of chemical behavior, make necessary the use by students of a specialized vocabulary than that of the general chemical. This includes the use of models, formulas, chemical equations and different meanings as alkanes, carboxylic acid, ester, etc.

- The language of chemistry is integral to the understanding of chemistry and the object program for ninth grade, allowing continuous enrichment of the language of chemistry.(Curricula and textbooks, applicated in modern teaching models. Tirana 2013)

\subsection{Social Sciences}

- Investigate the social changes in time in the community and forecast trends in the family, at work and patterns 


\section{of amusement}

- Review of the achievements in non-European nations and the perspectives of indigenous people for colonization.

- Comparison of different projections of the world maps and analyzes their attitudes and value.

- Forecast of economic, social and environmental issues in the light of demographic trends at national and immigration policy.

- Review of basic concepts of human rights through development and use of codes of rights and responsibilities in the schoolroom.

- Using the techniques of group and couples for conflict resolution in the classroom and school community. (Curricula and textbooks,, separate modern teaching models. Tirana 2013)

- Analysis of consequences and long-term psychological, societal and environmental conflicts war.

- Increase awareness of personal prejudices and fight against racism, gender and homophobia through challenging attitudes dispassionate and persuasive

- Planning and guiding action in the community to better the quality of life of people who are coping with psychological and mental challenges.

- Simulations review major global issues like developments that hold into account natural heritage and continuity, the population and the distribution of wealth, basic health care and the use of adult females.

- In the line of singing it can be done with: language (interpreting and making comments under topics that have songs), physical education (combination of physical movements with the music that they spill the beans). (Curricula and textbooks,, separate modern teaching models. Tirana 2013)

\subsection{Cross-curricular aspects are given in the sections of the program expansion.}

During its implementation, special care should be yielded to:

- Environmental issues, pollution and its protection;

- The refinement of communication (enabling students to read and utilize the information written in everyday life; (Integrated Curricula, ideas, challenges, suggestions)

- $\quad$ Media as the mass media, the Internet;

- The role of information technologies, audio-visual tools, data processor;

- Utilization of a foreign speech;

- Education for human rights;

- Matters of gender equality, ethnic, cultural, racial, spiritual;

- Patriotism (information cultural - artistic, economic, social, historical, geographical);

- Globalization

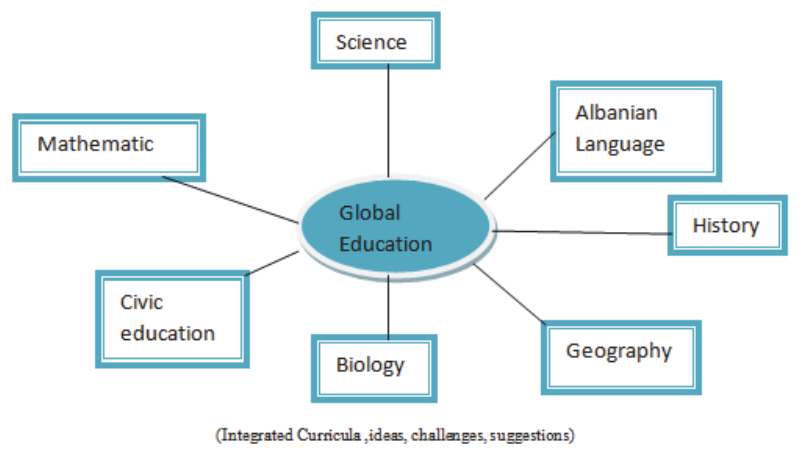

\section{What is the nation that currently occupies integration programs subject compulsory subject?}

How can the connection between the object of geography and citizenship education be realized

1. First, geography lessons should be an integral part of the citizenship dimension or "civic trend". They should refer to the concepts of citizenship, such as justice and human rights, and include bodily functions such as 
discussions etc.

2. Secondly, the size of citizenship during the topics of civic education should: To be a key issue of learning; It is linked to civic education curriculum.

\subsection{History and Civic Education}

First, this can be accomplished through the inclusion of issues, facts, events, etc. treated in the teaching of history in the curriculum of civic education.

The subject of history and that of civic education have in common:

1. Concepts: for example the power, authority, law, government, representation, liberty, etc.

2. Skills and habits, for example description, analysis, interpretation, discussion, research, interpretation, etc.

So, the connection between them can be reached through the following components

- Concepts: The characteristics of concepts (such as justice, equality, freedom, and so on) should be explained in time and space, so in concrete historical contexts, in the past and in the present.

- Report: In some lessons can be noted, dimensions of citizenship, for exemplar, in matters of human rights.

- Reach curriculum, continuous review of such topics as democracy, etc., throughout the cycle of schooling.

- Local dimension history should help increase citizen participation in community life, for instance, by undertaking projects that might include different generations.

\subsection{Examples of implementation of global education through the consolidation}

\subsubsection{Population}

- Research and graphical display of the history of the world's population increase and possible alternative developments (history, math)

- Identify the location of countries more and less populated in the world and compare different rates of population growth (geography, mathematics)

- Exploring the relationship between declining birth rate (add) and the improvement of living standards and health in developed nations and developing rural areas (history, social science)

- Review of birth control methods and comparing their effectiveness (social sciences, family, and science)

- Introduction to cultural factors, religious ones, etc. Oppose birth control and their potential impact on the population of the world (social science)

- Recognition (exploration) with arguments about the rights of women to settle for themselves fertility and fortune to their physical structure (social sciences, family)

- Reading the literature by women for abuse and sexual freedom (literature, social skills, and so on)

- Involvement in simulations and role plays about issues related to population (voice communications, humanities, social sciences)

- Analysis of the relationship between gender and power in school, family, residential district and country (social skill)

\subsubsection{Geography and civic training (Curricula, ideas, challenges, suggestions )}

1. What is common between Geography and Civic Education? They have in common:

- Concepts: human rights, community, interdependence, etc.,

- Skills: analysis, research etc.

- Content: The world as a global community, the challenges of global interdependence, and so forth

- Topics: housing, communication and so on

- Teaching methods, examination-based learning, research on specific events, and so on.

2. How can I help the case of geography in addressing the issues of civil training:

(Integrated Curricula ,ideas, challenges, suggestions)

- Help the students to better infer the impact of economic activity on people and the community in which they exist; 
- Highlights the relationship between local developments, internal and worldwide;

- Highlights the power of citizens to influence in the community and beyond;

- Reviews in width and in depth matters of sustainable growth;

- Encourages respect for other people and cultures and the surroundings.

3. How does the case of civic education help in developing curricula for geography or art?

Civic education: (Integrated Curricula ,ideas, challenges, suggestions)

- Uncovers links the subjects of geography course to deal with the everyday lives of pupils and increases their interest to them;

- Helps students realize the grandness of the discipline of geography;

- Furthers understanding of subjects linked to development, for example, just deal;

- Encourages pupils to participate in diverse development projects in the communities where they exist, for instance, on environmental matters.

\subsubsection{Racism}

- Reflecting on personal biases and the shock of being treated unfairly (social skills, humanities, and so on)

- Review the history of racism through case studies about racial intolerance in the world (history, social science)

- Use the game with roles and activities that are established along with the experience to develop a sensory faculty of empathy and intellect of the issues of racial discrimination (language, reading, social science)

- Use of internal statistical data on the impact of cultural descent, cultural and religious, racist attitudes (mathematics, etc.)

- Investigation / hate campaigns of hate campaigns in the local community and develop positive habits that under the power of persuasive arguments, challenge prejudicial remarks and behavior (social science, art, oral communication)

- Analysis of claims for genetic or hereditary superiority of single race over another (social science, skill, and so on)

- Exploring the connections between racial discrimination, sexuality, homophobia and cruelty towards other species (social skill)

- Evaluation of art, etc.

- Review study of the story of the several provinces and compare their stances on certain subjects (history, social sciences, and so on)

- Forecast the future of impact on the Albanian society and the impact of current policy in the immigration process.

\subsubsection{Science and civic training}

Today's order, constantly poses us questions related to our lives as individual programs as well as social programs. Management and resolution of these events often involves not only scientific knowledge, but also skills and habits to engage in moral discussions and particularly to make decisive assessment.

What are the similarities between science and citizenship?

- Ethical dilemmas that accompany decisions and research, for example,"can use animals to perform research"?

- Several topics about science and the environment, for instance, the subject of global warming; consumption of energy resources, genetically altered foods, cloning of animals and people etc.

- Impact of technical advances in society, for instance, the impingement of the Internet;

$>$ The study of civic education should serve pupils: (Integrated Curricula ,ideas, challenges, suggestions)

- Ponder and discuss the social implications of scientific developments;

- Assess the key purpose of science in the universe today;

- Understand the contradictory role of science and the fact that scientists have different and conflicting opinions as to the facts used as well as risks arising from scientific developments.

As understood from the above cases, the topic of connectivity curricular integration is to find itself in all curricular policies of the Institute of Curriculum and Standards. 
In the examples above it is hard to distinguish a single example of consolidation. In many elements seen integration of curricular models presented in a higher place.

Increased experience in the area of curriculum integration will increase the spaces and possibilities for the execution of other integration models. The mixture of different exercise rules will add positive and at the same time will reduce the disadvantages of each particular model.

Today it is widely accepted and estimated that there will come a time that students will expose for themselves the interrelatedness and interdependence that exists in the material world. But, alas, the facts indicate that reading them today depends on what we instruct. If we teach students interconnection and integration, they learn to understand matters in this light, if we teach class and lack of continuity in a fourth dimension of things, they learn to see things off the connection and mutual interdependence and outside history their development in time, so even given once and for all.

The revised curriculum outlines three pedagogical principles related to the bodily function and catching methods, integrated curriculum and environment that supports scholarship. They met it in the process of learning:

- The feeling of surprise and the natural wonder of the child is the main ingredient that motivates learning;

- The child is an active factor in the process of learning;

- Learning is a developer in his nature;

- The experience and existing knowledge of the child form the basis of learning;

- The child's current environment provides the context for learning;

- Learning involves leading activity and methods;

- Language is central to the learning process;

- The child may benefit from the artistic dimension of scholarship;

- Social and emotional dimensions are significant components of learning;

- Learning is most efficient when it is incorporated;

- Develop critical thinking skills and problem resolving;

- Learning process is characterized by cooperative learning;

- Evaluation is taken in as an intact function of teaching and scholarship.

\subsection{The Difference between traditional curricula and competency-based}

It concentrates on inputs

It concentrates on teachers and subjects

Faculty member

Split up into subjects and instructed according to them

The rigid in construction and on time dispersion

Exclusively guiding and Local autonomy

in the centralized system

Concentrating on the needs of "scholastic"

The methodology gives priority participating and interoperability

It focuses on assessments generalizing

It gives precedence to the methodology outside the lecture hall

It gives precedence to the theoretical perspective
It focuses on yield and results of teaching / Scholarship.

It focuses on scholars

The methodology set between wide field of learningand cross-curricular

Flexible in structure and saving time

Allows flexibility based on different stages and in different cases within certain principles and quality measures

Open to the community and the lifelong learning

Assists intellectual skills of a high level at the same time of prefabricated knowledgeto the publicity and the advancement of social, skills, emotional, spiritual, technical, practical, motor art, and so on

It focuses on the format and content Progress

The methodology gives priority participating and interoperability

The methodology gives priority complex through theory which is connected with practice, application and solving problems 
The methodology gives priority conformist. Care and educates creativity

It gives priority to uniformity learning Individualization of care learning

Comparison between the two curricula shows that curriculum changes are necessary for the evolution of society by acquiring a competence-based pedagogy.(Pedagogical magazine - "ER" "Education Review)

\subsubsection{Curricular reform priorities.}

Established on the demands of reality and the underlying trends in the world of education, the current curriculum reform in our country singled out as a key priority, long-term:

- Possessing a high stage of speech.

- Possessing high level math.

- Possessing two foreign languages.

- Training and learning technology entrepreneur

\subsection{Conclusions and recommendations}

Curriculum that is shaping our country is based on educational philosophies that take into account the following principles:

- Full and harmonious development of the child,

- Importance of evaluating individual changes

- The importance of natural action and detection methods

- Integrated nature of the course of study

- Importance of the environment that supports scholarship

- Increased opportunity to act, for individual work, group, project, task, essay, poetry Internet searches, and so on

- Through situations that promotes teacher, teaching becomes more interesting for students, and learning extends beyond knowledge of the textbook to their implementation in routine life.

- Parents can cooperate more to help their children in common projects.

- Learning the powers of pupils enables more about life through virtual and concrete learning. Generalization of practical situations makes theoretical knowledge with stable turning in skills, values and attitudes.

- This way of teaching creates greater freedom for the planning, organization of the class and the teaching plan.

- The curriculum in comparison with that existing charge is less for the age of pupils.

\subsubsection{Curriculum reform in the future should expand the autonomy and accountability of the school, consisting of:}

- Enriching the curriculum in accordance with the nature of the schools, enabling more effective and fruitful learning process at a given time;

- Adapting the curriculum to the needs of diverse scholars.

- Individualization of instruction;

- Creating opportunities for additional paths, suitable and desirable, for certain groups of scholars;

- The creation of spaces that, in addition to the core curriculum, the programs or modules include cases related to education of children with exceptional needs;

- Active involvement of schools in a continuous process of improving teaching experiences, increasing their responsibility for increasing student achievement;

- Active involvement of schools and local education units in a continuous process of collecting and analyzing data, documenting the challenges and solutions towards meeting the requirements of the official curriculum.

- Must be drafted new textbooks which comply fully with the new curriculum program, as teachers of schools where the curriculum is implemented it has encountered difficulty in drafting lesson plans from the program mismatching existing textbooks.

- The program of new curriculum should be clearer for teachers in terms of themes and topics distribution 
under the new program.

- To develop a long-term strategy priority curricular short move forward;

- Design a dynamic plan for the implementation of new curricula and continuing improvement;

- To develop a strategy for public awareness and participation;

- To create mechanisms for institutional capacity and human resource development;

- The envisaged steps to implement the curriculum with three entry points in the first grade, sixth and tenth;

- To implement the reform of alternative texts;

- To implement new training program for teachers;

- To implement the new program for managing and improving schools.

- Creating opportunities for additional courses, suitable and desirable for certain groups of students;

- The creation of spaces that, in addition to the core curriculum, the programs or modules include subjects related to education of children with special needs;

- The active involvement of schools and local education units in a continuous process of collecting and analyzing data.

4.4.2 At the end of lowsecondary education, the pupil:

- Understands, develops and communicates ideas and information in their mother tongue.

- Reads, writes, listens and communicates various kinds of texts (literary, non-literary and media ), in accordance with the purposes and specific audiences.

- Owns basic knowledge of the natural sciences and society and the importance of practical value.

- Owns and uses appropriate methods and skills to live, learn and work in the school, family and community.

- Understands him as part of a group or society and adapt to different situations arising in the family, school and community.

- Communicate effectively, using different means and methods of communication.

- Use different methods for solving problems and making decisions argues his election.

- Distinguishes features of contexts (geographic, cultural and historical) and uses them in everyday life relationships.

- Assesses the cultural heritage of the Albanian people and respects other nations.

- Use technology equipment at home, school and community.

- He feels the need to deal with different physical activities and sports and conducts such optional activities

- It preserves and develops personal health and actively helps in maintaining the health of others

- Applies knowledge and skills in creative and different circumstances.

- He protects his rights and those of others.

- Builds relationships with others without prejudice, on the basis of mutual respect

- It possesses knowledge of the skills necessary for higher schooling levels

- Digital methods creates quality in teaching.

- Pupils feel equal to the evaluation for one another.

- Filing pupils work makes it more motivated

- The teacher has more actions and space in drafting the curriculum and curricular integration.

\section{References}

Curricula and textbooks, applicated in modern teaching models. (Tirana 2013)

Integrated Curricula (ideas, challenges, suggestions)

Pedagogical magazine "Education Review" Tirana 2014

Pedagogical magazine Tirana 2011 